\title{
OUTER DISC ROTATION FROM CEPHEID VARIABLES
}

\author{
FREDERIC PONT \\ AND \\ DIDIER QUELOZ, MICHEL MAYOR AND PIERRE BRATSCHI \\ Geneva Observatory \\ 1290 Sauverny \\ Switzerland
}

\begin{abstract}
We have measured radial velocities for a large number of classical cepheids of the Galaxy, particularly in the outer disc. We determine the rotation curve up to a galactocentric radius of $16 \mathrm{kpc}$. The results are then compared to similar studies using HII regions. We also consider some possible complications.
\end{abstract}

\section{Introduction}

Classical cepheids are bright, young stars, and both their distance and radial velocity are relatively easy to determine. This makes them ideal probes of the rotation of the Galaxy.

We present here the results of two studies of the Galactic rotation curve using classical cepheids :

- The first from a survey of faint cepheids (up to V=12 mag at $6<$ $R<12 \mathrm{kpc}$ ) with the CORAVEL spectrometer at ESO (Chile), begun in 1983 and completed last year (Pont et al. 1994a,b). The aim was to get a new determination of the local rotation constants $A, R_{0}, u_{0}, v_{0}$ as well as to have a closer look at some characteristics of the local velocity field.

- The second study consists of a sample of about 30 very faint cepheids of the outer disc (up to $\mathrm{V}=15 \mathrm{mag}, R>11 \mathrm{kpc}$ ) measured in radial velocities with CORAVEL and the ELODIE spectrometer of OHP (France). This program began in early 1994 and is ongoing. Its aim is to determine the rotation curve beyond $\mathrm{R}=11 \mathrm{kpc}$, a range in which the currently most reliable values, mainly from HII region data, are not very clear, showing a dip around $R=10 \mathrm{kpc}$ followed by an ambiguous rise at large $R$. 


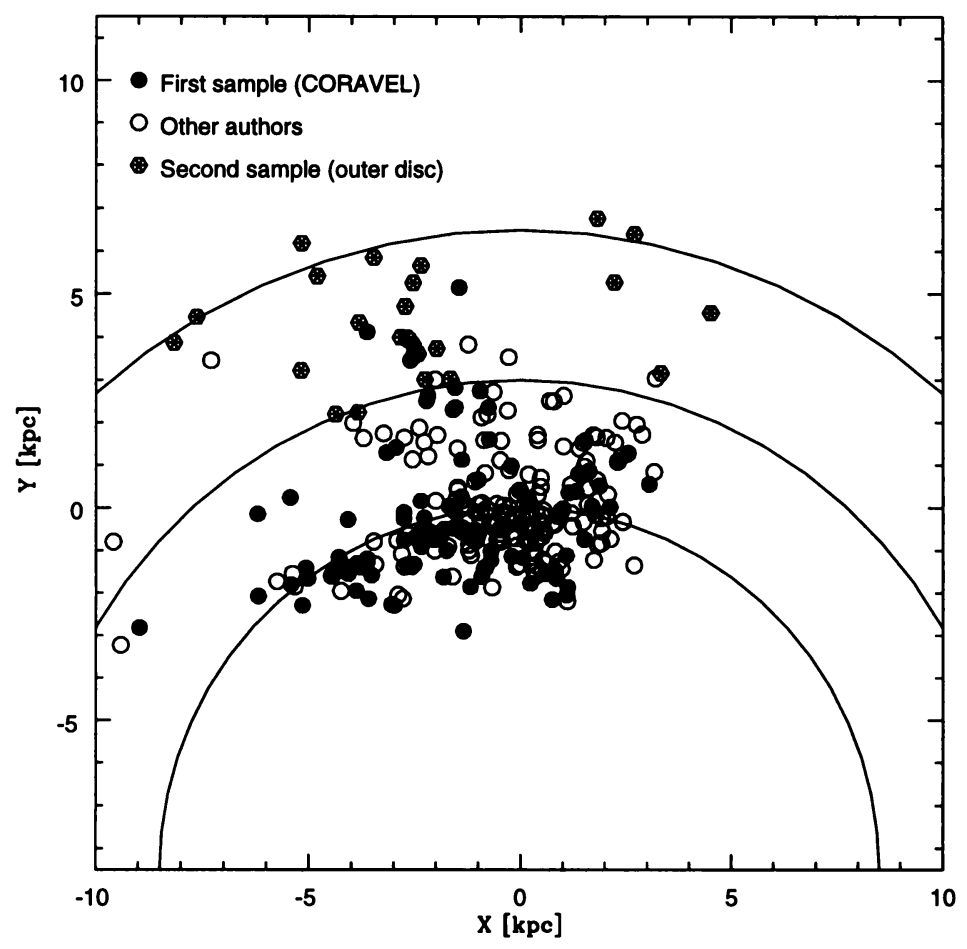

Figure 1. Position of the classical cepheids with known $\gamma$-velocities projected onto the galactic plane. The Sun is at $(0,0)$, the galactic centre at $(0,-8.5)$. Circles are drawn at $\mathrm{R}=8.5,11.5$ and $15 \mathrm{kpc}$. "Other authors" are mainly : Joy (1937), Stibbs (1956), Metzger et al. (1992), Moffett \& Barnes (1987).

\section{Observational Data}

In order to use a population of stars as kinematical tracers, accurate 'distance, radial velocity' pairs are needed. Radial velocities were gathered with ELODIE or CORAVEL, and BVI photometry was complemented from La Silla (Chile) and Pic du Midi (France), or kindly communicated by Arne Henden before publication. Cepheid distances were computed from BVI photometry using period-luminosity-colour relations from Feast \& Walker (1987) and Caldwell \& Coulson (1987), and reddenings following Fernie (1990). Fig. 1 shows the position in the galactic plane of the considered set of classical cepheids with known $\gamma$-velocities. Notice that the outer disc sample does reach much remoter parts of the disc.

Our strategy, in order to obtain centre-of-mass radial velocities (" $\gamma$ velocities") from radial velocity pulsation curves with a minimum number of observations, was to get 5-6 measurements for every cepheid, evenly spread 
over the pulsation phase, then to fit the data with the velocity curve of another cepheid of similar period. With this procedure one can recover the $\gamma$-velocity within $2-3 \mathrm{~km} \mathrm{~s}^{-1}$, a sufficient precision for galactic kinematics since the uncertainties are dominated by the $\sim 10 \mathrm{~km} \mathrm{~s}^{-1}$ intrinsic velocity dispersion.

\section{Results}

The main results are the followings :

- First, using an axisymmetric rotation model, we obtained a new set of rotation constants : $R_{0}=8.09 \pm 0.30 \mathrm{kpc}, A=15.92 \pm 0.34 \mathrm{~km} \mathrm{~s}^{-1} \mathrm{kpc}^{-1}$ and $2 A R_{0}=257 \pm 7 \mathrm{~km} \mathrm{~s}^{-1}$.

- The rotation curve is well constrained by classical cepheids between 6 and $11 \mathrm{kpc}$.

Our data also offer strong evidence that the $\sim 3 \mathrm{~km} \mathrm{~s}^{-1}$ mean residual (between observed and expected velocities) long observed with cepheids is indeed a real dynamical effect and not, as sometimes proposed, an intrinsic shift between measured and actual $\gamma$-velocity (Pont et al. 1994a).

- The outer disc sample brings a strong constraint on the rotation velocity for $11<R<15 \mathrm{kpc}$. Using the standard values $R=8.5$ and $\theta_{0}=220 \mathrm{~km} \mathrm{~s}^{-1}$, the rotation curve beyond $R=11 \mathrm{kpc}$ remains flat at the $\sim 200 \mathrm{~km} \mathrm{~s}^{-1}$ level (Fig. 2).

\section{Comparison with HII Region Data}

A comparison between Fig. 2 and Fig. 3, displaying the rotation curve for HII regions, makes it clear that the cepheid data show no evidence of the rotation velocity rise seen in the HII region data. It seems that this interesting discrepancy cannot be attributed to noise in the cepheid data, since the cepheids are quite tightly grouped around $v_{\text {rot }} \simeq 200 \mathrm{~km} \mathrm{~s}^{-1}$. Large errors on radial velocities are also unlikely.

Now, there could be some systematic shift in either cepheid distances or HII region distances. A distance scale change moves the points diagonally in the $v_{\text {rot }}$ vs. $R$ graphs (Figs. 2 and 3 ), the distance entering the calculation of both quantities. A better way to look at it is on the angular velocity $(\Omega)$ versus $R$ graph (Fig. 4 ). The advantage of such a representation is that distance scale changes move the points horizontally. In this diagram, the HII region data would be around the $240 \mathrm{~km} \mathrm{~s}^{-1}$ line at large $\mathrm{R}$. What is to be seen in Fig. 4 is that to bring the two sets of data in agreement by adjusting the distance scale, rather large distance changes are needed. Moreover, if the cepheids were to carry all the burden of this change, they would be brought to quite unreasonable distances $(14-20 \mathrm{kpc})$ which would 


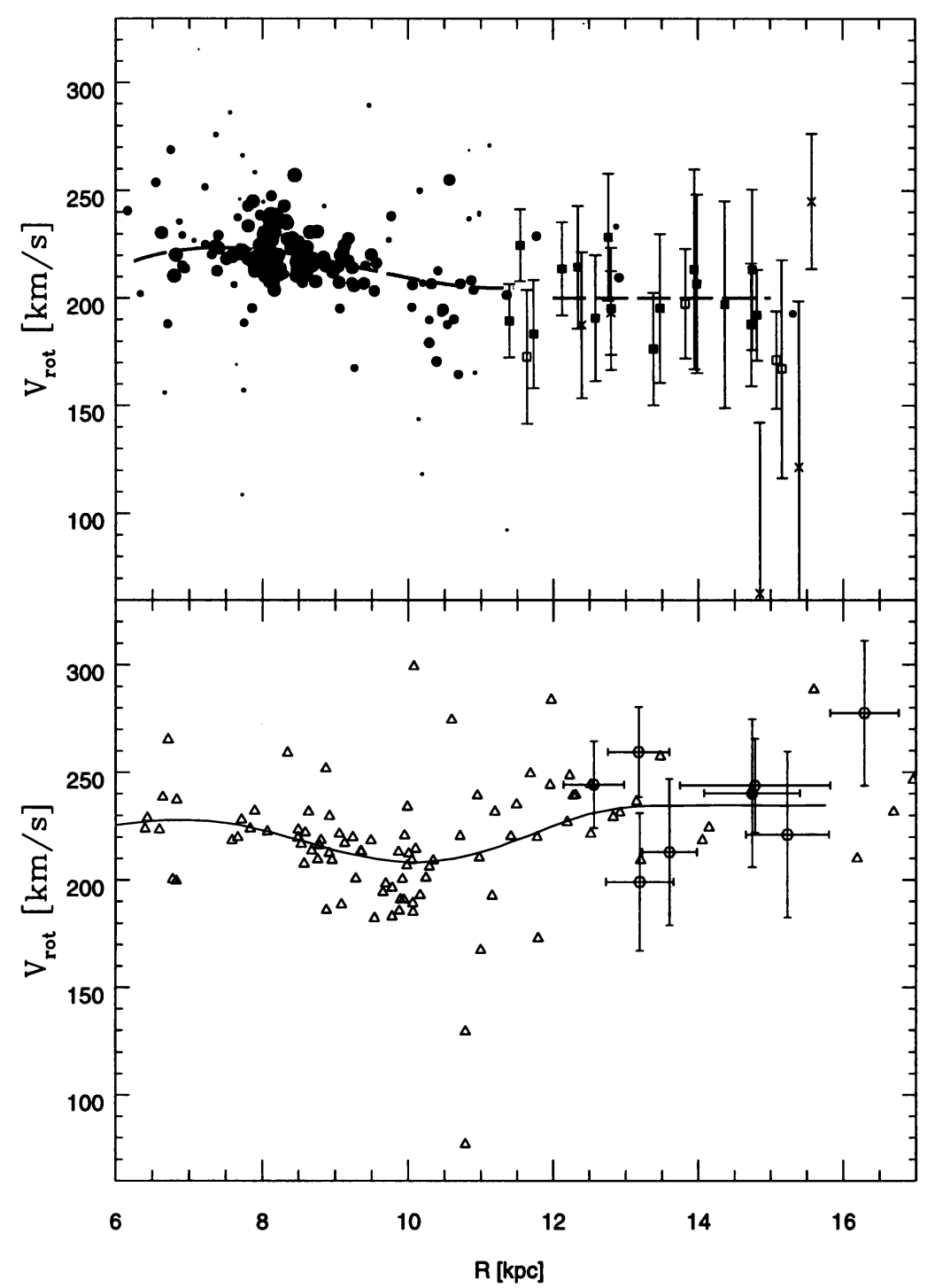

Figure 2. (top) Rotation curve for classical cepheids, assuming $R_{0}=8.5 \mathrm{kpc}$ and $\theta_{0}=+220 \mathrm{~km} \mathrm{~s}^{-1}$. For the first sample, the dots have a size proportional to their weight in the fit (a function of galactic longitude mainly). The cepheids of the second sample are plotted as black squares, white squares if their photometry is incomplete, and crosses for possible type II cepheids. Error bars correspond to a $10 \mathrm{~km} \mathrm{~s}^{-1}$ kinematical velocity dispersion on the line-of-sight. The dashed line indicates the rotation curve fitted with an axisymmetric rotation model for $6<R<11.5 \mathrm{kpc}$ and a flat curve at $200 \mathrm{~km} \mathrm{~s}^{-1}$ for $R>12 \mathrm{kpc}$.

Figure 3. (bottom) Rotation curve for HII regions. Data collected from Fich et al. 1989 (triangles), Turbide \& Moffat 1993 (hexagons with error bars) and Clemens 1985 (curve) on the same scale as Fig. 2. Note that while the cepheid data remains flat at $200 \mathrm{~km} \mathrm{~s}^{-1}$, the HII region data rises up to $v_{\text {rot }} \simeq 240 \mathrm{~km} \mathrm{~s}^{-1}$ or higher. 
make their positions in the galactic plane rather unrealistic. Thus, it seems that either the rotation velocity of cepheids and HII region is different (different rotation velocity or different non-circular motion), or the HII region distance scale has to be somehow shortened.

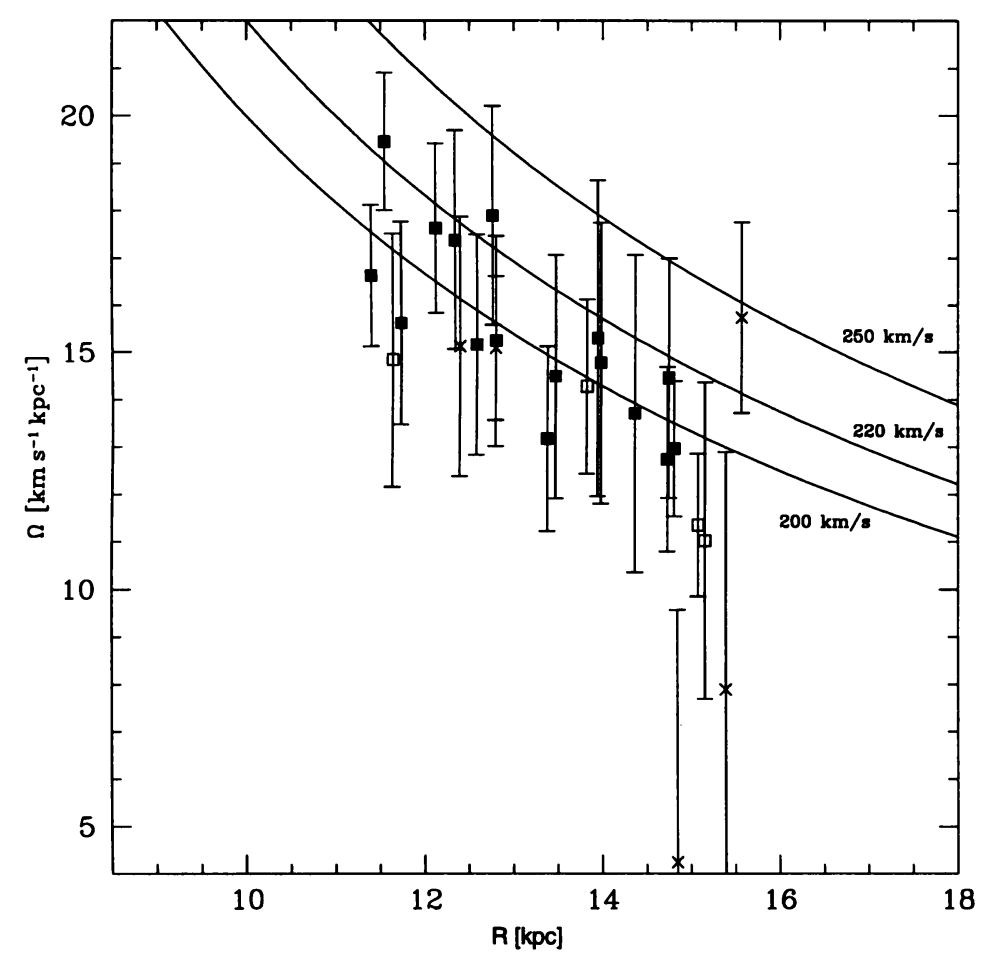

Figure 4. Angular velocity curve, $\Omega$ vs. $R$, for outer disc cepheids. Three lines at constant $v_{\text {rot }}$ are indicated. Same symbols as in Fig. 2. The HII region data would be around $v_{\text {rot }}=240 \mathrm{~km} \mathrm{~s}^{-1}$ for $R>13 \mathrm{kpc}$. In this graph a distance scale change moves the points horizontally. Notice that bringing the cepheid data to $v_{\text {rot }}=240 \mathrm{~km} \mathrm{~s}^{-1}$ would push many of them beyond $R=16 \mathrm{kpc}$.

\section{Difficulties for $R>11 \mathrm{kpc}$ Cepheids}

\section{Type II Cepheids}

The most daunting problem with the outer disc cepheid sample is the possible presence of type II cepheids. For a variety of reasons, type II cepheids are even more difficult to discriminate in the outer disc than near the Sun. However, the dispersion of the final $\gamma$-velocities around that predicted by a flat rotation curve at $200 \mathrm{~km} \mathrm{~s}^{-1}$ is only $\sigma\left(v_{o b s}-v_{\text {model }}\right)=8 \mathrm{~km} \mathrm{~s}^{-1}$. This value is even lower than the velocity dispersion of solar neighbour- 
hood cepheids $\left(\sim 10 \mathrm{~km} \mathrm{~s}^{-1}\right)$, and much lower than that expected for type II cepheids $\left(\sigma_{\text {typeII }}>20-30 \mathrm{~km} \mathrm{~s}^{-1}+\right.$ scatter from inaccurate distances). Thus, we feel confident in expecting only a small number of type II cepheids in our sample.

\section{Non-axisymmetric Motions}

Any determination of the rotation curve from radial velocities without proper motions is vulnerable to non-axisymmetric motions, not included in the model. At first sight, the northern and southern data show no difference, which puts limits on a LSR radial motion for instance. But the sample is not large enough at present to be more specific.

\section{Metallicity Corrections}

If there is a metallicity gradient in the disc, one expects high $R$ cepheids to be metal deficient. This affects the distance determination in two ways : it changes the coefficients of the PL or PLC relations, and it changes the reddening determinations for BVI photometry.

We used theoretical magnitudes and colours from Chiosi et al. (1993) to predict the changes introduced by metal deficiency on the cepheid distance determination. We also considered corrections proposed by Caldwell $\&$ Coulson $(1985,1987)$ for comparison.

It appears that the agreement between $\mathrm{PL}(\mathrm{V}), \mathrm{PLC}(\mathrm{BV})$ and $\mathrm{PL}(\mathrm{I})$ distances is much lowered by the corrections. More specifically, something seems to be wrong with the reddening correction. To discriminate between the possible causes of this discrepancy (insufficient photometry, lower gradient in the disc, inaccurate theoretical colours, changes in $\mathcal{R}, .$. ), we intend to get more photometry, and a spectroscopic indication of metallicity, for the outer disc cepheids.

\section{References}

Caldwell J. \& Coulson I. 1985, MNRAS 212, 879

Caldwell J. \& Coulson I. 1987, AJ 93, 1090

Chiosi C., Wood P., Capitanio N. 1993, ApJSS 86, 541

Feast M. \& Walker A. 1987, ARAA 25, 345

Fernie J. 1990, ApJSS 72, 153

Fich M., Blitz L., Stark A. 1989, ApJ 342, 272

Joy A. 1937, ApJ 86, 363

Metzger M., Caldwell J., Schechter P. 1992, AJ 103, 529

Moffett T., Barnes T. 1987, PASP 99, 1206

Pont F., Mayor M., Burki G. 1994a, A\&A 285, 415

Pont F., Burki G., Mayor M. 1994b, A\&AS 105, 165

Stibbs D. 1956, MNRAS 116, 453

Turbide L. \& Moffat A. 1993, AJ 105, 1831 


\section{DISCUSSION}

Feast: Do you see any evidence of streaming motions ?

Pont: The residual velocity field exhibits local wavy fluctuations of the order of $5 \mathrm{~km} / \mathrm{s}$ on a typical scale of $2 \mathrm{kpc}$, but with no obvious pattern. As for the outer disc sample, the north-south symmetry is good.

H. van Woerden: Could the difference of rotation curves between HII regions and Cepheids be caused by a difference in non-axisymmetric motions, related to a difference in age? A difference of 50-100 Myr would suffice.

Pont: In principle it could I suppose. The cepheids are still young, but there may have been enough time for them to escape the gas. streaming motions. However, the HII regions studied cover a large portion of the outer disc, so that rather large-scale non-axisymmetric motions would be needed to explain the difference. If this is the case, a good knowledge of the cepheid rotation curve is important, since for most application one is interested in the stellar rotation curve. 